\title{
Reply to 'Eukaryote lateral gene transfer is Lamarckian'
}

Roger reply - Martin argues here ${ }^{1}$ and elsewhere ${ }^{2}$ that nearly all claims of lateral gene transfer (LGT) into eukaryotic genomes are untrue, and that accompanying evolutionary narratives are fundamentally 'Lamarckian'. Some eukaryote LGT claims have proved false, but this does not mean that most are. Although rare, gene transfers have had a profound effect on the evolution of traits in eukaryotes ${ }^{3}$.

Mechanisms potentially mediating eukaryote LGT are well-documented and include natural transformation ${ }^{4}$, interdomain conjugation ${ }^{5}$, cell fusion ${ }^{6}$, viral transduction $^{7}$ and DNA acquisition from endosymbionts $^{3,8}$. Eukaryotic genomes are littered with remnants of viruses and transposons, both of which have been shown to move between different eukaryotic species $^{7,9}$, sometimes mobilizing host genes. DNA from bacterial endosymbionts has been inserted into dozens of insect and nematode host chromosomes ${ }^{8}$; many of these cases have been confirmed by rigorous follow-up experiments.

Most pieces of acquired DNA are probably useless. Sometimes, however, new genes confer novel capacities. For example, in the gut parasites Blastocystis spp., several acquired genes were identified that are otherwise only present in bacteria (spliceosomal introns are present, ruling out bacterial contamination) ${ }^{10}$. Closely related homologues in enteric bacteria are involved in host immune system evasion. Other than LGT, what other reasonable explanations are there for the origins of these genes in Blastocystis? They cannot be ancestral to all eukaryotes and differentially lost — what would the last eukaryotic common ancestor have done with genes to defend against a host that had not yet evolved? Similar arguments can be made for the hundreds of documented ancient and recent LGTs that allow diverse eukaryotes to defend against pathogens, acquire nutrients, adapt to new environments or parasitize hosts ${ }^{3,6}$.
What about the charge of Lamarckism? It is groundless. Prokaryote and eukaryote LGT narratives have exactly the same form and invoke precisely the same neoDarwinian mechanism for adaptation. Chunks of DNA are accidentally incorporated into chromosomes creating genetic variation that is neutral, deleterious or, in rare cases, beneficial. If they enhance fitness, acquired genes are likely to be fixed in the population by natural selection. Any reasonable adaptive LGT claim has a similar etiological narrative that respects modern evolutionary principles.

Is this Lamarckian? No. Like other mutational mechanisms, LGT processes are not directed by their potential fitness effects. Furthermore, acquired genes are not phenotypic characters of organisms that are modified by habits of use; only the latter are relevant to Lamarck's original ideas ${ }^{11}$. One study reinterprets Lamarckism in light of molecular genetics ${ }^{12}$, arguing that LGT in both prokaryotes and eukaryotes constitutes a form of 'quasi-Lamarckian' evolution. The authors suggest that environments are enriched in genes 'adaptive' for life in that environment by virtue of the organisms that inhabit them. An organism entering this environment therefore has a higher chance (relative to another environment) to sample an 'adaptive' gene by LGT. However, even in this account, the outcome of LGT is still probabilistic with respect to fitness. Rather than being Lamarckian, this phenomenon is better seen as the biased availability of mutations causing directional evolution ${ }^{13}$.

So why such resistance to LGT in eukaryotes? Endosymbiotic organelle origins and endosymbiotic gene transfer have been championed as dominant mechanisms in eukaryotic genome evolution ${ }^{2}$. Indeed, the widely publicized 'hydrogen hypothesis' of eukaryogenesis depends heavily on assuming a mitochondrial ancestry of 'bacterial-like' enzymes of anaerobic energy metabolism in eukaryotes ${ }^{2}$. Acknowledging
LGT as an important mechanism provides an alternative explanation for such patchily distributed genes in eukaryote genomes that do not show the hallmarks of mitochondrial or plastid origin. But LGT complicates accounts of eukaryote evolution, making it difficult to infer the metabolisms of ancient endosymbionts and determine their roles in eukaryogenesis. As biologists, we must embrace this complexity, because that is life.

\section{Andrew J. Roger}

Centre for Comparative Genomics and Evolutionary Bioinformatics, Department of Biochemistry and Molecular Biology, Dalhousie University, Halifax, Nova Scotia, Canada.

e-mail:andrew.roger@dal.ca

Published online: 13 March 2018

https://doi.org/10.1038/s41559-018-0522-6

References

1. Martin, W. F. Nat. Ecol. Evol. https://doi.org/10.1038/s41559-0180521-7 (2018).

2. Martin, W. F. BioEssays 39, 1700115 (2017)

3. Husnik, F. \& McCutcheon, J. P. Nat. Rev. Microbiol. 16, 67-79 (2018)

4. Nevoigt, E., Fassbender, A. \& Stahl, U. Yeast 16, 1107-1110 (2000).

5. Lacroix, B. \& Citovsky, V. mBio 7, e00863-16 (2016).

6. Soanes, D. \& Richards, T. A. Annu. Rev. Phytopathol. 52, 583-614 (2014).

7. Filée, J. Virology 466-467, 53-59 (2014).

8. Dunning Hotopp, J. C. D. et al. Science 317, 1753-1756 (2007).

9. Panaud, O. C. R. Biol. 339, 296-299 (2016).

10. Eme, L., Gentekaki, E., Curtis, B., Archibald, J. M. \& Roger, A. J. Curr. Biol. 27, 807-820 (2017).

11. Burkhardt, R. W. Genetics 194, 793-805 (2013).

12. Koonin, E. V. \& Wolf, Y. I. Biol. Direct 4, 42 (2009).

13. Stoltzfus, A. \& Yampolsky, L. Y. J. Hered. 100, 637-647 (2009).

\section{Acknowledgements}

I am grateful to A. Simpson, M. Leger, M. O’Malley, J. McCutcheon, T. Richards, F. Doolittle, L. Eme and A. Inkpen for discussion and critical review of the text. Financial support for this work comes from the Canada Research Chairs programme, the Natural Sciences and Engineering Research Council and the Canadian Institutes of Health Research.

Competing interests

The author declares no competing interests. 